\title{
INEQUALITY OF THE PUPILS IN EPILEPTICS, WITH A NOTE ON LATENT ANISOCORIA.'
}

By WILLIAM BROXNNING, M.D., BRookLYN, N. Y.

$\mathrm{T}$

$\mathrm{HE}$ condition of inequality of the pupils, technically termed anisocoria, is not very rare even in persons of average health. No great importance attaches to it in any class of troubles, but as an objective sign it is worthy of further study.

Its occasional occurrence in epilepsy has long been noticed, and a diagnostic value has even been attributed to it in suspected cases of the nocturnal form (Fürstner, I886).

It is of course merely a symptom, occurring casually where there happens to be some implication, if only exhaustion, of the pupillary paths or centres.

In a series of I 50 consecutive cases of epilepsy observed for interval symptoms at the Long Island Hospital Dispensary in the last eight years, this condition was noted altogether in $16,{ }^{2}$ although varying some in degree and constancy. Of these 16 cases, 12 were males and 4 females (of the whole series of I50, 84 were males and 66 females). Only $3\left(\frac{3}{16}\right)$ were in patients over 22 years, while two-fifths $\left(\frac{2}{5}\right)$ of the whole series were over that age. One of these three older patients was a typical Jacksonian, another was probably some secondary form of convulsions, and the third showed slight indications of tabes: hence it is evident that in idiopathic epilepsy, anisocoria occurs predominantly among the younger patients.

A few quotations from authorities at hand will serve for comparison.

Schleick (I886), in examining I27 hospital epileptics, found only one case of decided difference in the width of the pupils, the eyes being otherwise normal. Marie (Arch. de

'Presented to the American Neurological Association, Washington, D. C., Sept. 22, 23 and 24,189 I.

2 In one other case it was questionable, but put down as negative. In still another, the outline of one pupil may not have been quite regular. 
Neurolg., I882, Paris) observed an inequality in 8 of 53 institution cases (about $15 \%$ ), the difference amounting to $\frac{1}{3} \mathrm{~mm}$. in 6 and to $\frac{2}{3} \mathrm{~mm}$. in 2. Musso ( 1884 , quoted by Hare) found that there was in $22.8 \%$ (of 70 cases) an inequality of the pupils. Addison ( 1867 , also from Hare) found an inequality in 2 of 50 insane epileptics. Oliver, of Philadelphia, from his studies on 50 adult American male epileptics at the Norristown Asylum (1887), says: "Pupils are, as a rule, equal in size and alike in shape."

As to the proportion in non-epileptics, or as to what proportion of the above cases may be due to local anomalies of the eye itself, we have no figures for comparison.

The different proportions found by the above authorities may be partly due to closeness as well as time of observation. For, in frequent cases, there is just a suggestion of inequality, yet not so pronounced as to be at first unmistakable. Moreover, the condition seems to be somewhat more frequent and more marked directly after an attack. The different age of the patients, as already indicated, may have some influence.

Probably the above proportion in my own cases, of 16 in 150 , might have been increased had the slighter inequalities been more carefully sought, especially in the earlier cases of the series. But a close scrutiny even of this limited number suffices to show certain important differences, and to suggest more exactly why there may readily be a wide diversity in such statistics.

Three somewhat distinct types are distinguishable.

I. Where there is a very considerable inequality.

This form-doubtless the only one heeded by many observers ${ }^{3}$-occurred in a special class of patients. There were three such cases, and each was in an epileptic with decidedly unilateral symptoms, These were all in younger males, though of course this form might well occur at any age.

One of the three was a syphilitic with typical Jacksonian seizures. The second was probably hereditarily syph-

${ }^{3}$ As, e.g., Schleick, Addison and Oliver, mentioned above. 
ilitic (when finally relieved of his epilepsy the pupils gradually became equal). The third patient had suffered a traumatic cerebral hemorrhage as a cause.

These cases show that any great inequality of the pupil in an epileptic depends on some localized intracranial trouble, $i . e$., it is then not so much an epileptic as a focal symptom.

Of course it goes without saying that in plenty of cases of unilateral epilepsy there is no anisocoria.

2. Cases of slight inequality, and in which the condition is fairly constant.

This does not preclude some variation from one observation to a subsequent one. In a few cases the immediate effect of the seizures is noticeable, but even then it is rather to accentuate the condition than to develop it anew each time.

Of this form there were ten examples, indicating that it is more frequent in epileptics than the other two forms combined. In certain of these the difference was so trifling that the observation had to be independently corroborated before its acceptance. Doubtless in these cases the peculiarity is only functional. It might simply be classed as an exhaustion-sign, like the pareses seen at times after seizures, but.for its greater persistence when once present. Sometimes in these first two forms the wider pupil was observed tore-actless than the other, indicating (according to Heddæus) that then the inequality was due to sphincterparesis of the wider pupil.*

3. Latent anisocoria-distinct inequality only in faint illumination.

Various non-epileptic cases first convinced me that there is a frequent form of latent pupillary inequality-pos-

4 Dr. James Oliver (Brain, October, I888, p. 359) says : "When the pupils are unequal [in epileptics], the last effort at accommodation may determine equality, the inequality only reappearing with the state of comparative rest. In testing the reaction to light, I have frequently remarked that, whilst the pupils may, in conjunction, react well to this excitation, separately the one more dilated will be found to contract with less certainty and less markedly than the smaller, and at the same time, manifests a greater tendency to return forthwith to its pre-existing state of dilatation." 
sibly not well recognized as yet-and that a more exact method of examination and specification might be adopted.

These were cases of astigmatism, brain-tumor, old apoplexy with atrophy of one optic nerve (the most striking case of all), various other nerve troubles, and also individuals otherwise apparently normal. In these there was a more or less marked inequality during faint or poor illumination, but such inequality quite disappeared on strongly and generally illuminating both eyes.

Dr. J. C. Shaw also tells me of a case in a syphilitic ataxic in whom with scant illumination there was irregular outline as well as inequality of pupils, both abnormal conditions disappearing completely on exposure to strong diffused light.

To guard against the frequently deceitful effects of shadows, if lateral illumination be used in examining for slight degrees of this form, it should be tried alike from each side before deciding.

As a plausible explanation it may be suggested that whilst the passive (i.e., sympathetic) innervation of the pupils is in such cases unequal or disproportionate, the reflex impulse-equal for the two eyes-is, when fully called into play, quantitively so far in excess as to completely overbear all passive ones and so for the time being to wholly dictate the pupillary condition. The relative superiority of the oculomotor control of the pupil, as compared with the sympathetic, is of course a matter of every-day observation.

This explanation interprets the morbid phenomenon as a symptom resulting not simply from bilaterally uneven sympathetic action, but as one that only appears at times when the action of the oculomotor is relatively or absolutely in abeyance.

Hence we should distinguish :

(1) A passive or latent anisocoria.

(2) An active or at least continuous in equality.

If possible, the type observed should always be specified. Of course the first two forms of inequality above described belong to the second of these classes. 
Certainly, even with this precaution, it remains a relative matter so far as estimating the degree of illumination -and in some of these latent cases the visual power was greatly reduced in one or both eyes. The minimum limit is, however, furnished by the least illumination consistent with a proper inspection of the pupil.

Although most of these observations were in other troubles, this form was demonstrable in 2 of the last 50 epileptics; and a recent examination of an earlier case (still an epileptic) discloses a marked example of this. Doubtless, an ignoring of this matter in making examinations might give a certain diversity of results, although as a whole, an exclusively latent inequality is evidently not very common in these patients.

As a rule, including the epileptic cases, the inequality even in least illumination was very slight; but in one case (non-epileptic), it was as great as almost ever seen from natural causes.

Of the three classes of cases, it can confidently be asserted that in the present series, all those of the first class have been noted, and hence for this type they may serve as a proper average ( $\mathrm{I}$ in 50 ).

But at first, little attention was paid to the second and third classes. If, however, only the last 50 cases in the series be considered, it transpires that amongst these, pupillary inequality occurred 8 times (those of the first form all happened to occur in the first 100 cases). This gives $16 \%$ and agrees very well with that of Marie $(15 \%)$ or that of Musso $(22.8 \%)$. Together, these give 32 cases of anisocoria in 173 cases of epilepsy, or about $18.5 \%$. In other words, it appears that on an average one epileptic in every five or six will, if examined with care, prove to have some, though usually slight, inequality of the pupils. As this conclusion is based on the fairly harmonious results of three observers in different countries, it is admissible to infer that those finding a smaller proportion must have simply overlooked the common slighter difference in size of the pupils. 